\title{
Article
}

\section{Equation to Line the Borders of the Folding-Unfolding Transition Diagram of Lysozyme}

Mohammad, Mohammad Amin, Grimsey, Ian M. and Forbes, Robert Thomas

Available at https://clok.uclan.ac.uk/15857/

Mohammad, Mohammad Amin, Grimsey, Ian M. and Forbes, Robert Thomas orcid iconORCID: 0000-0003-3521-4386 (2016) Equation to Line the Borders of the Folding-Unfolding Transition Diagram of Lysozyme. Journal of Physical Chemistry B, 120 (28). pp. 6911-6916. ISSN 1520-6106

It is advisable to refer to the publisher's version if you intend to cite from the work. http://dx.doi.org/10.1021/acs.jpcb.6b01317

For more information about UCLan's research in this area go to http://www.uclan.ac.uk/researchgroups/ and search for <name of research Group>.

For information about Research generally at UCLan please go to http://www.uclan.ac.uk/research/

All outputs in CLoK are protected by Intellectual Property Rights law, including Copyright law. Copyright, IPR and Moral Rights for the works on this site are retained by the individual authors and/or other copyright owners. Terms and conditions for use of this material are defined in the policies page.

\section{CLoK}

Central Lancashire online Knowledge www.clok.uclan.ac.uk

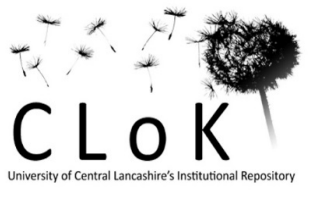


$4{ }^{\dagger}$ Drug Delivery Group, School of Pharmacy, University of Bradford, Bradford BD7 1DP, UK.

$5 \quad \ddagger$ Department of Pharmaceutics, Faculty of Pharmacy, University of Damascus, Damascus, Syria.

6 §School of Pharmacy and Biological Sciences, University of Central Lancashire, PR12HE, UK.

$8 *$ Corresponding author

9 Dr Mohammad Amin Mohammad

10 Associate Professor in Pharmaceutical Technology

11 First name: Mohammad Amin

12 Family name: Mohammad

13 Phone: + $44(0) 1225386797$

14 Email: mam2014uk@gmail.com

15 Postal address: Dr. Mohammad Amin Mohammad, School of Pharmacy, University of Bradford,

16 Bradford, West Yorkshire, BD7 1DP, UK.

Dr Ian M. Grimsey

19 Senior Lecturer in Pharmaceutical Technology

20 Phone: $+44(0) 1274234754$

21 Fax: +44 (0)1274 234769

22 i.m.grimsey@bradford.ac.uk

23 School of Pharmacy

24 University of Bradford

25 Bradford, West Yorkshire, BD7 1DP.

Prof Robert T. Forbes

28 Professor of Clinical Pharmaceutics

29 Phone: +44 (0)1772 893513

30 rtforbes@uclan.ac.uk

31 School of Pharmacy and Biomedical Sciences

32 University of Central Lancashire

33 Preston, Lancashire, PR1 2HE. 
42 It is important for the formulators of biopharmaceuticals to predict the folding-unfolding transition

43 of proteins. This enables them to process proteins at predetermined conditions without 44 denaturation. Depending on apparent denaturation temperature $\left(T_{m}\right)$ of lysozyme, we have derived 45 an equation describing its folding-unfolding transition diagram. According to water content and 46 temperature, this diagram was divided into three different areas which are the area of the water-

47 folded lysozyme phase, the area of the water-folded lysozyme phase and the bulk water phase, and 48 the area of the denatured lysozyme phase. The amount of water content controlled the appearance 49 and intensity of Raman band at $\sim 1787 \mathrm{~cm}^{-1}$ when lysozyme powders were thermally denatured at 50 temperatures higher than $T_{m}$.

51

52

53

54

55

56

57

58

59

60

61

62 
Protein structure and dynamics are determined by the intraprotein and protein-water non-

66 covalent interactions. ${ }^{1,2}$ Hydration degree of proteins, which is the weight ratio of water to protein,

67 should be $\sim 0.2$ to initiate protein function, moreover, full protein function requires a hydration

68 degree of $\sim 1 .^{3}$ However, proteins are in their solid state during pharmaceutical processes and

69 storage. Water contents of protein powders affect their stability and performance. ${ }^{4}$ Water content

70 alters the mechanisms of proteins' degradation reactions, and this alteration also depends on other

71 conditions such as temperature and the formulation compositions. ${ }^{5}$ In general, increasing water

72 content decreases the stability of protein powders. Water acts as a plasticizer which enhances the

73 molecular mobility of the proteins and so leads to an increase in their degradation rates. This

74 shortens the shelf-life of solid protein powders and formulations.

75 Many studies have used lysozyme as a model protein to understand the effect of the water

76 content on protein stability using differential scanning calorimetry (DSC). Previous works used

77 sealed DSC pans to study the effect of water content on the glass transition temperature $\left(T_{g}\right)$ of

78 lysozyme, they found that $T_{g}$ of lysozyme decreased by increasing the water content to approach

79 a plateau level. ${ }^{6,7}$ Similarly, increasing water content within lysozyme powders decreased their

80 apparent denaturation temperature $\left(T_{m}\right)$ in sealed DSC pans. ${ }^{8}$ Therefore, both water content and

81 temperature line the borders between the native (folded) and denatured (unfolded) states of

82 lysozyme powders. These borders are important to predetermine the processing conditions (water

83 content and temperature) which preserve the native structure of proteins during manufacturing

84 processes (e.g., spray drying). Here, we derive an equation correlating water content of lysozyme

85 powders with their $T_{m} s$, and then use this equation to line the borders of the folding-unfolding 
transition diagram of lysozyme. Also, we use Raman spectroscopy to monitor water content effect

87 on the molecular conformation of lysozyme upon the thermal unfolding transition of lysozyme 88 powders.

EXPERIMENTAL METHODS

Materials. Lyophilized hen egg-white lysozyme powder (Biozyme Laboratories, UK) was

93 purchased and considered to be unprocessed lysozyme powder.

94 Sample Preparation. The unprocessed lysozyme powder was used to prepare a series of 95 lysozyme powders with different water contents either by raising or reducing their water content.

96 The water residues were manipulated by exposing the powders to humidified air or to anhydrous

97 nitrogen gas for different times at $30{ }^{\circ} \mathrm{C}$. The prepared powders were immediately sealed and left

98 to equilibrate at room temperature at least for a weak after preparation. This time is enough to 99 ensure uniformity of water distribution in lysozyme particles. ${ }^{9}$ The prepared series of lysozyme 100 powders were tested to correlate the water content with $T_{m}$. To monitor the water content effect 101 on the molecular conformation of lysozyme upon the thermal unfolding transition, the lysozyme 102 powders with different water contents were thermally denatured inside hermetically sealed pans 103 by heating to temperatures higher than their unfolding transition peaks. These samples were 104 considered to be the thermally denatured lysozyme powders.

105 Thermogravimetric analysis (TGA). The water content of each powder was determined 106 using Thermo Gravimetric Analysis (TGA) (Perkin Elmer Ltd., UK). Samples (5-10 mg) were 107 heated from $30{ }^{\circ} \mathrm{C}$ to $210^{\circ} \mathrm{C}$ at a scan rate of $10{ }^{\circ} \mathrm{C} / \mathrm{min}$ in an aluminum pan under nitrogen flow 108 at $20 \mathrm{ml} / \mathrm{min}$. Each sample was analyzed in triplicate. The decrease in the weight before 
109 decomposition was considered as water content. TGA results were validated by re-analyzing the 110 water content of some samples using Karl Fischer Titration (KFT) (701 KF Titrino with 703 Ti 111 stand, Metrohm, Switzerland). Using TGA instead of KFT as only a few mg is enough for TGA.

112 Differential scanning calorimetry (DSC). $T_{m}$ of each sample was measured using 113 Differential Scanning Calorimetry (DSC) (Perkin-Elmer Ltd., UK). To study the effect of water 114 residue on the thermal stability of lysozyme (the value of $T_{m}$ ), the evaporation of water during 115 heating was suppressed by using Large Volume Capsules, which are stainless steel sample 116 containers equipped with an O-ring seal to provide a hermetic seal. Lysozyme powders (5-10mg) 117 and solutions in water $(20 \mu \mathrm{l})$ were encapsulated in the hermetically sealed pans and scanned 118 between $40{ }^{\circ} \mathrm{C}$ to $200{ }^{\circ} \mathrm{C}$ at $5{ }^{\circ} \mathrm{C} / \mathrm{min}$ in triplicate. DSC was also used to prepare the thermally 119 denatured lysozyme powders by heating them inside the hermetically sealed pans to temperatures 120 higher than their unfolding transitions.

121 FT-Raman spectroscopy. FT-Raman spectra of the lysozyme powders at different water 122 content and their thermally denatured lysozyme counterpart powders were recorded with a Bruker 123 IFS66 optics system using a Bruker FRA 106 Raman module. The excitation source was an Nd: 124 YAG laser operating at $1064 \mathrm{~nm}$ and a laser power of $50 \mathrm{~mW}$ was used. The FT-Raman module 125 is equipped with a liquid nitrogen cooled germanium diode detector with an extended spectrum 126 band width covering the wave number range $1850-1100 \mathrm{~cm}^{-1}$. Samples were placed in stainless 127 steel sample cups and scanned 200 times with the resolution set at $8 \mathrm{~cm}^{-1}$. The observed band wave 128 numbers were calibrated against the internal laser frequency and are correct to better than $\pm 1 \mathrm{~cm}^{-}$

129 . The spectra were corrected for instrument response. The experiments were run at a controlled 130 room temperature of $20 \pm 1^{\circ} \mathrm{C}$. 


\section{Results and discussion}

We employed this protein because it folds in a highly cooperative manner and so exhibits

135 an all-or-none thermal unfolding transition, and so it displayed one denaturation peak, in solid and

136 solution states, ${ }^{10,11}$ which makes the study easier. The driest prepared lysozyme powder contained

$1372.6 \pm 0.1 \% \mathrm{w} / \mathrm{w}$ water and its $T_{m}$ was $436.9 \pm 0.6 \mathrm{~K}$, while the wettest powder contained $20.3 \pm 2.3$

$138 \%$ w/w water and its $T_{m}$ was $355.5 \pm 3.1 \mathrm{~K}$. In Figure 1 is a DSC thermogram of each sample, 139 showing an inverse correlation between water content and $T_{m} \mathrm{~s}$.

140

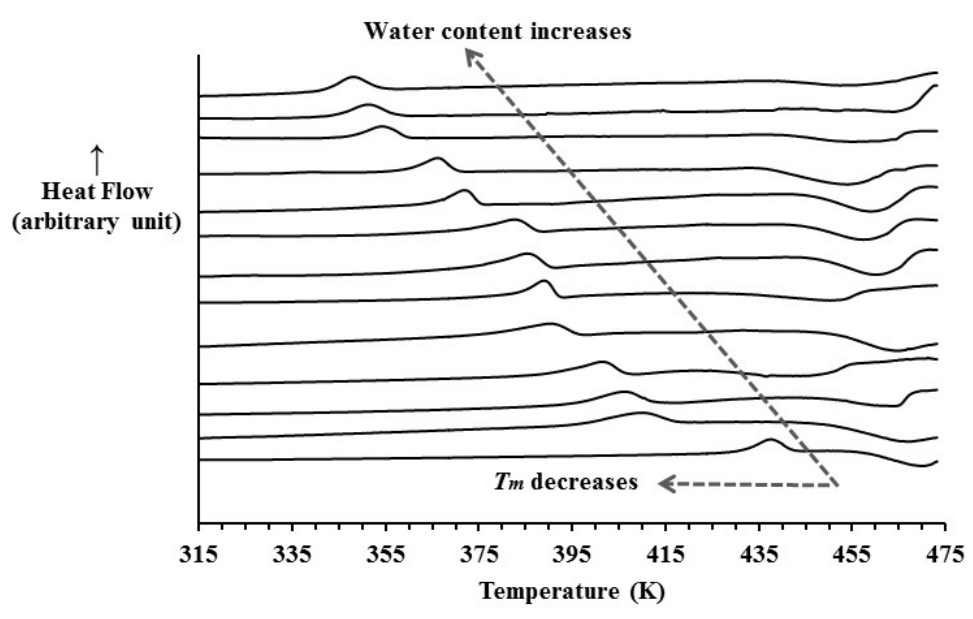

Figure 1. Differential scanning calorimetry thermograms of lysozyme powders (in sealed pans) at different water content.

143 water content performed in pierced pans showed two endothermic peaks, the first broad peak,

144 which extends from 298 to $433 \mathrm{~K}$, indicates water evaporation and its area directly correlates to

145 the water content, the second peak at $\sim 473 \mathrm{~K}$ refers to the protein denaturation $\left(T_{m}\right)$ and its position 
146 is independent of water content because water evaporates through the pierced pans before the 147 unfolding transition (denaturation) of lysozyme. ${ }^{10}$

148 However, unlike the experiments performed in the pierced pan, Figure 1 shows that the 149 position of the endothermic unfolding peak $\left(T_{m}\right)$ varied for different water contents when DSC 150 experiments were performed under sealed conditions. The DSC thermograms (Figure 1) did not

151 show the water evaporation peak because the evaporation of water during heating was suppressed 152 by the hermetical seal, i.e., the pressure generated from increasing the temperature of the air, which 153 had been trapped during the capsulation of the hermetically sealed pans, prevented water loss. 154 Figure 1 also show an exothermic peak after lysozyme unfolding in all samples analysed with the 155 sealed pans. This peak was reproducible and showed little variation between samples, with a peak 156 position ranging from 453 to $468 \mathrm{~K}$. It was believed that this exothermic peak could be due to the 157 aggregation of lysozyme molecules after unfolding, however re-scanning the sealed pans 158 containing previously aggregated lysozyme powders also gave an exothermic peak at the same 159 position but without the endothermic unfolding peak.

160 We hypothesized that water molecules associate with lysozyme powders to form a 161 homogenous water-folded lysozyme phase. This phase unfolds at a kinetic thermal energy 162 depending on water mole fraction $\left(X_{w}\right)$ and lysozyme mole fraction $\left(X_{p}\right)$ within the powders. When $163 X_{w}$ increases, $T_{m}$ of the unfolding kinetic energy is reduced, and its reduction stops when the water 164 molecules reach their saturation limit within the water-folded lysozyme phase. Above this limit, 165 water molecules segregate from the water-folded lysozyme phase to form another phase of bulk 166 water, and so they cannot reduce $T_{m}$ further.

$167 \quad X_{w} / X_{p}$ equals $\mathrm{N}$, the ratio of water molecules to lysozyme molecules, and it is calculated 168 according to Eq. (1): 


$$
N=14296 \times \mathrm{W} \% / 18 \times \mathrm{P} \%
$$

17018 and 14296 are the molecular weights of water and lysozyme, respectively, and W\% and P\% are

171 the percentage of water weight and protein weight in the powders, respectively. The results were 172 tabulated in Table 1.

173

Table 1. The apparent denaturation temperature $\left(T_{m}\right)$, number of water molecules associating with one lysozyme molecule $(N)$, water mole fraction $\left(X_{w}\right)$ and lysozyme mole fraction $\left(X_{p}\right)$ of lysozyme powders at different water content.

\begin{tabular}{ccccc} 
Water content \%w/w & $T_{m}(\mathrm{~K})$ & $N$ & $X_{w}$ & $X_{p}$ \\
\hline $2.5(0.1)$ & $436.9(0.6)$ & 20.4 & 0.9532 & 0.0468 \\
$4.6(0.3)$ & $412.0(1.6)$ & 38.1 & 0.9744 & 0.0256 \\
$5.2(0.1)$ & $406.2(2.1)$ & 43.4 & 0.9775 & 0.0225 \\
$6.4(0.2)$ & $401.9(1.4)$ & 54.3 & 0.9819 & 0.0181 \\
$7.7(0.1)$ & $390.3(0.2)$ & 66.6 & 0.9852 & 0.0148 \\
$8.5(0.1)$ & $388.8(2.6)$ & 73.8 & 0.9866 & 0.0134 \\
$9.2(0.5)$ & $385.5(0.3)$ & 80.5 & 0.9877 & 0.0123 \\
$9.9(0.1)$ & $383.5(0.6)$ & 87.7 & 0.9887 & 0.0113 \\
$12.0(0.1)$ & $371.8(0.4)$ & 108.7 & 0.9909 & 0.0091 \\
$14.4(0.1)$ & $365.7(0.4)$ & 133.1 & 0.9925 & 0.0075 \\
$15.6(0.6)$ & $364.0(1.6)$ & 146.6 & 0.9932 & 0.0068 \\
$20.3(2.3)$ & $355.5(3.1)$ & 201.8 & 0.9951 & 0.0049 \\
\hline
\end{tabular}

Values within parenthesis are standard deviation, $\mathrm{n}=3$.

$175 T_{m}$ reflects the value of thermal kinetic energy needed to overcome the cohesion energy 176 stabilizing the folded state of proteins. This cohesion energy results from Van der Waals attraction, 177 electrostatic interaction, hydrogen bonding, and conformational entropy (hydrophobic effect). ${ }^{12}$

178 Electrostatic and hydrophobic interactions play the main roles in protein folding. ${ }^{1,13}$ Water affects 179 these interactions and hence the magnitude of $T_{m}$ needed to unfold proteins. Water molecules 180 surround the surface of protein molecules and penetrate into their cavities. ${ }^{14}$ Surrounding water 181 molecules strengthen the hydrophobic effect by an increase in the entropy of water due to 182 association between hydrophobic residues. ${ }^{13}$ However, according to Coulomb’s law, the interior 
183 waters weaken the electrostatic forces because they increase the dielectric constant in the interior

184 of protein molecules and also increase the distances between polarized and ionized groups of

185 proteins. ${ }^{15,16}$ Our results demonstrated that the net effect of water weakens the forces responsible

186 for the folded state, and so the increased water content facilitates the protein unfolding at lower

$187 T_{m}$. Dehydration of lysozyme powders stabilizes its native form because the free energy change of

188 the lysozyme denaturation increases by dehydration. ${ }^{17}$

189 Multiplying the gas constant $(R)$ by $T_{m}$ gives the kinetic thermal energy needed to unfold

190 lysozyme. Interestingly, our data (Table 1) prove that $R T_{m}$ and $\ln N$ correlate linearly with a

191 correlation coefficient close to one $(r=-0.997)$, and their linear equation is:

$$
\ln N=\text { Slope } R T_{m}+\text { Intercept }
$$

193 Figure 2 shows the straight line of Eq. (2) which was regressed in Eq. (3).

$$
\ln N=-\frac{R T_{m}}{72.3}+15.0
$$

195 where 72.3 has units of Cal.mole $\mathrm{e}^{-1}$ and 15.0 has units of mole.mole ${ }^{-1}$ (i.e., unitless). $R=1.9872041$

196 Cal.mole ${ }^{-1} \cdot \mathrm{K}^{-1}$, therefore Eq. (3) can be reduced to Eq. (4):

$$
T_{m}=-36.4 \ln N+545.7
$$

198

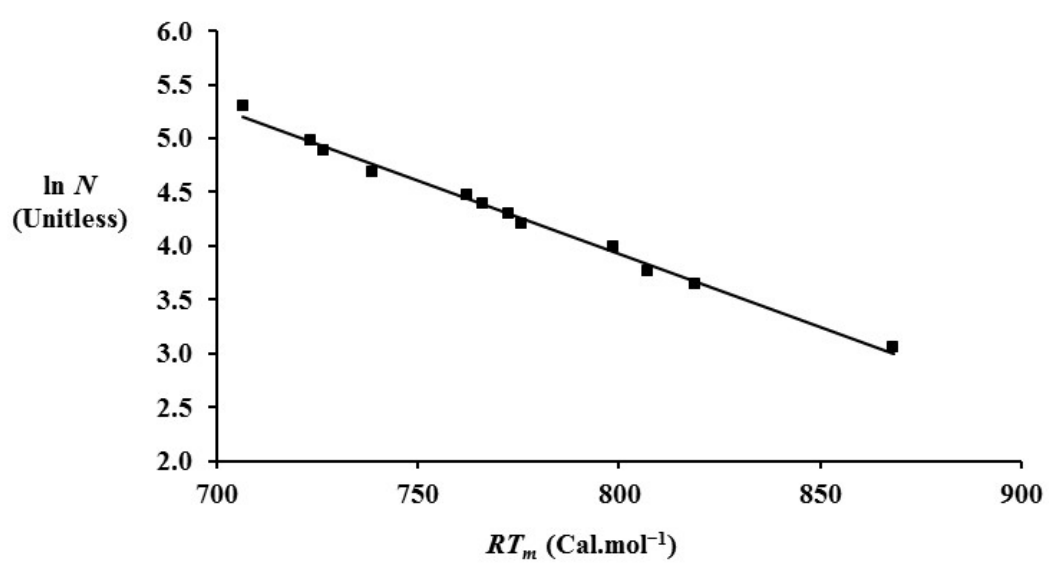

Figure 2. The linear correlation between the denaturation thermal kinetic energy and logarithm of water molecules associating with one lysozyme molecule. 
200 of the solutions were $348.2 \pm 0.7$ and $348.0 \pm 1.0 \mathrm{~K}$, respectively, and they were similar (T-test: P <

201 0.05). According to our hypothesis, $T_{m}$ of the lysozyme solutions equals the apparent denaturation 202 temperature of the saturated water-lysozyme phase $\left(T_{m}^{S}\right)$. Therefore, we extrapolated Eq. (4) to $T_{m}^{S}$

203 (348K), which is the same as that of the solutions, to calculate the number of water molecules 204 which saturate one molecule of lysozyme $\left(N^{S}\right)$ as follows:

$$
T_{m}^{S}=-36.4 \ln N^{S}+545.7
$$

206 The calculated $N^{S}$ was 228 water molecules according to Eq. (5). This water amount is equivalent 207 to $22.3 \% \mathrm{w} / \mathrm{w}$ and $0.3 \mathrm{~g} / \mathrm{g}$ protein. We then prepared another lysozyme powder with a water content 208 of $23.2 \pm 1.1 \% \mathrm{w} / \mathrm{w}$, which is similar to the calculated $N^{S} . T_{m}$ of the prepared water saturated 209 powder was $347.8 \pm 1.4 \mathrm{~K}$, which is similar to $T_{m}^{S}$ (T-test: $\mathrm{P}<0.05$ ). Therefore, we used temperature 210 and $\mathrm{N}$ to draw the folding-unfolding transition diagram of lysozyme. Figure 3 shows this diagram

211 in which the denaturation line obeys Eq. (3) until reaching the water saturated solid state at $N^{S}$. 212 After this value of water molecules, $T_{m}$ did not significantly change. Our calculated value of $N^{S}$, 213 i.e. 228 water molecules, is similar to previously reported values of the hydration shell of 214 lysozyme. Previous researchers used gigahertz to terahertz spectroscopy ${ }^{18,19}$ Raman 215 spectroscopy ${ }^{20}$ neutron and X-ray scattering ${ }^{21} \mathrm{NMR}^{22}$ to quantitate the hydration shell of 216 lysozyme, and these methods estimated similar values of the hydration shell of lysozyme, and the 217 values ranged between 170-270 water molecules. Hydration shell molecules have different 218 properties from bulk water. ${ }^{23}$ Our results demonstrate that the effect of water molecules on $T_{m}$ 219 changed at $N>N^{S}$. Therefore, we speculate that water molecules begin to form the bulk water at $220 N>N^{S}$. Therefore, the folding-unfolding transition diagram of lysozyme (As shown in Figure 3) 221 was divided to three areas; (A) represents the area of the water-folded lysozyme phase, (B) 
222 represents the area of the water-folded lysozyme phase and the bulk water phase, and (C)

223 represents the area of the denatured lysozyme phase, and the line separating (C) form (A) and (B)

224 is the denaturation line.

225

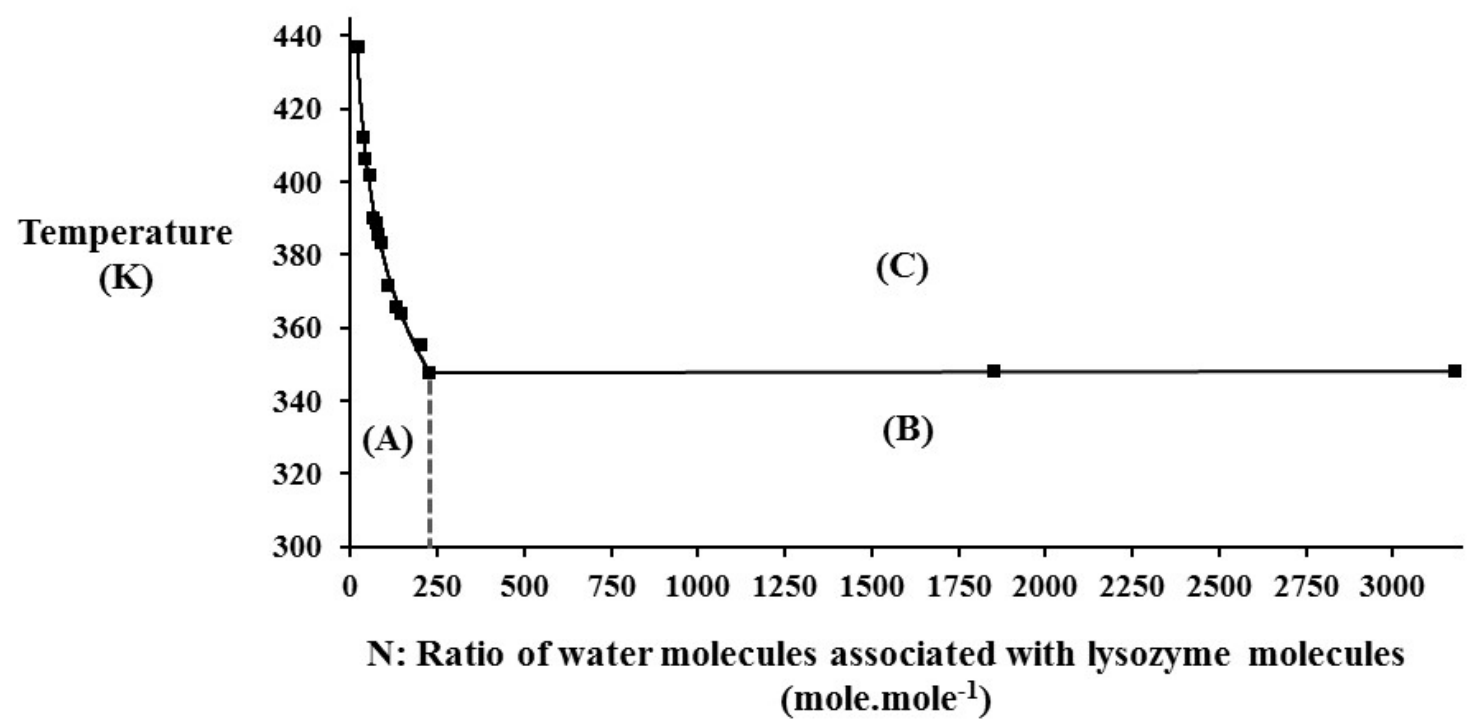

Figure 3. The folding-unfolding transition diagram of lysozyme. (A) represents the area of the water-folded lysozyme phase, (B) represents the area of the water-folded lysozyme phase and the bulk water phase, and (C) represents the area of the denatured lysozyme phase; the line separating (C) form (A) and (B) is the denaturation line.

Although $T_{m}$ of lysozyme solutions is usually $348 \mathrm{~K}$, it changes due to additives (e.g., co-

228 solvents, $\mathrm{pH}$, ionic strength). ${ }^{10,24,25}$ Also, additives changed $T_{m}$ of lysozyme powders. ${ }^{8}$ We could

229 conclude that if the additives are miscible with water, they will participate in forming water-protein

230 phases (in both solid and solution states). Therefore, they would change the constants of Eqs. (3)

231 and (5). Whilst other proteins may have different constants.

232 Previous Raman study clarified the significant effects of hydration degree of native 233 lysozyme powders on the back bone and the side chain conformations of lysozyme. ${ }^{9}$ In other 234 words, this previous study covered the molecular conformation of lysozyme under the denaturation 
235 line of the folding-unfolding transition diagram of lysozyme. Here, the molecular conformation of

236 lysozyme above the denaturation line were investigated. Raman spectra of the thermally denatured

237 powders at different hydration degree and their original powders were collected at room

238 temperature and presented in Figure 4. The peak intensities of the spectra were normalized using

239 the band at $1447 \mathrm{~cm}^{-1}$ (CH bending) as internal intensity standard. This is because its intensity and

240 position are unaffected by changes induced in protein structure after applying different stresses. ${ }^{26}$

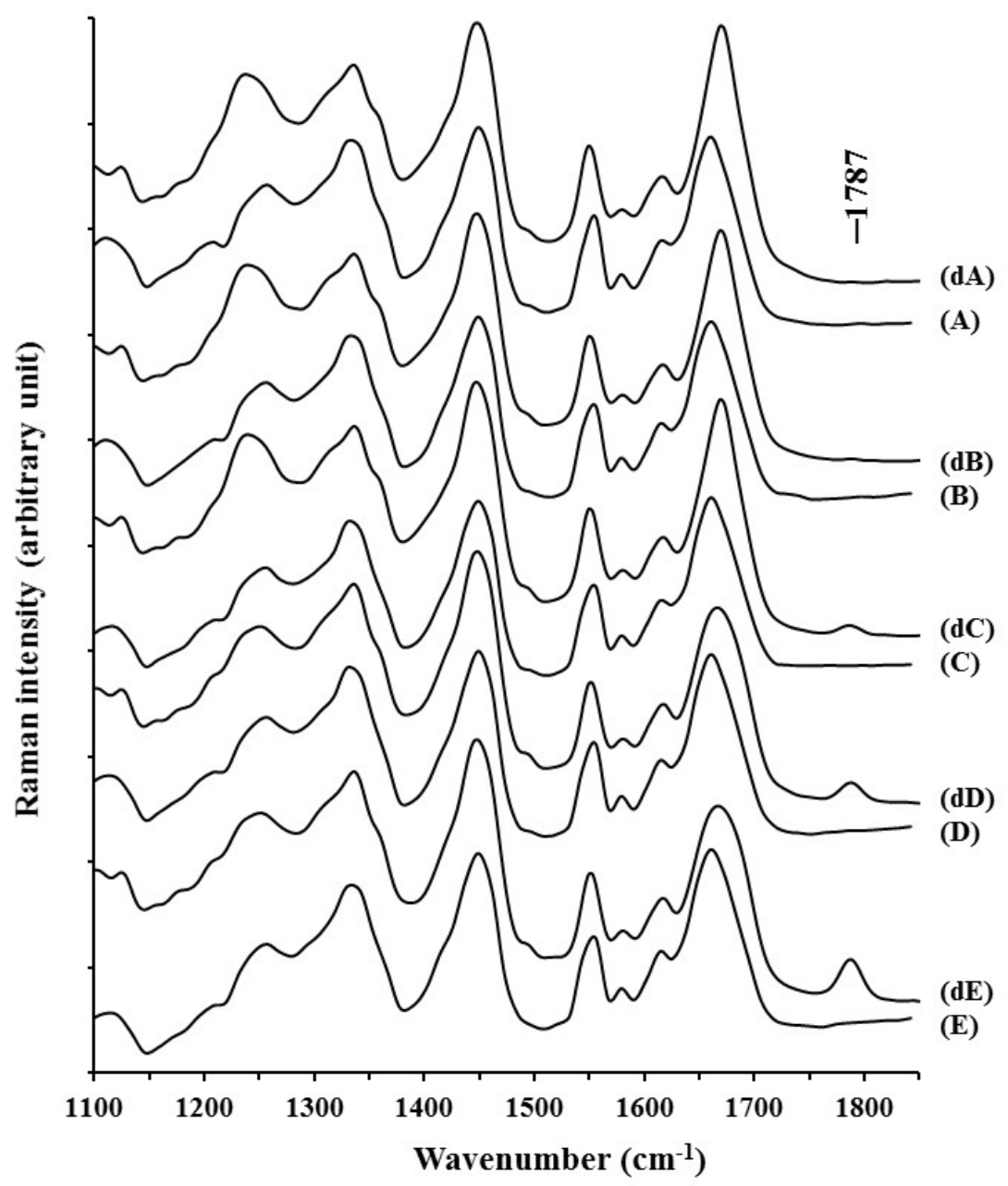

Figure 4. Representative Raman spectra of lysozyme powders in the $1100-1850 \mathrm{~cm}^{-1}$ wavenumber region, (A), (B), (C), (D) and (E) represent lysozyme powders at different water content of 20.3, 12.0, 8.2, 5.2 and $2.5 \% \mathrm{w} / \mathrm{w}$, respectively, and (dA), (dB), (dC), (dD) and (dE) represent their thermally denatured counterpart powders (in sealed pans), respectively. 
243 significant differences between the studied original lysozyme samples (Figure 4, spectra A, B, C,

244 D and E). However, compared to the original lysozyme powders, the Raman spectra of the

245 counterpart thermally denatured powders showed changes in the the peak positions of amide I and

246 amide III bands. The peak of amide I was upshifted from $\sim 1660$ to $\sim 1671 \mathrm{~cm}^{-1}$ after thermal

247 denaturation (Figure 4, spectra $\mathrm{dA}, \mathrm{dB}, \mathrm{dC}, \mathrm{dD}$ and $\mathrm{dE}$ ), and also spectra $\mathrm{dD}$ and $\mathrm{dE}$ show 248 broadening in the amide I band in the case of the thermally denatured samples at low hydration 249 degree (5.2 and $2.5 \%$ w/w). The peak of amide III was downshifted from $\sim 1256$ to $\sim 1249 \mathrm{~cm}^{-1}$ at 250 low hydration degree (spectra $\mathrm{dD}$ and $\mathrm{dE}$ ), and it was further downshift to $\sim 1240 \mathrm{~cm}^{-1}$ with an 251 increase in the intensity at higher hydration degree (spectra dA, dB and dC). The Raman Bands of 252 the amide I ( $\mathrm{C}=\mathrm{O}$ stretch) and amide III ( $\mathrm{N}-\mathrm{H}$ in-plane bend $+\mathrm{C}-\mathrm{N}$ stretch) are indicators of the 253 protein’s secondary structures. ${ }^{9}$ The mid-point of amide I is expected to be at $1665 \pm 5 \mathrm{~cm}^{-1}$ for $\alpha$ 254 helix and $1670 \pm 3 \mathrm{~cm}^{-1}$ for $\beta$-sheet and disordered secondary structure, and the mid-point of amide 255 III is expected to be at $1267 \pm 8 \mathrm{~cm}^{-1}$ for $\alpha$-helix, $1235 \pm 5 \mathrm{~cm}^{-1}$ for $\beta$-sheet and $1245 \pm 4 \mathrm{~cm}^{-1}$ for 256 disordered structure. ${ }^{27,28}$ Therefore, the changes in Raman bands (presented in Figure 4) indicate 257 that the content of the $\beta$-sheet and disordered secondary structure were increased and at the same 258 time the $\alpha$-helix content was decreased after the thermal denaturation. Similar changes in the 259 positions of amide I and amide III Raman bands of lysozyme powders were reported when 260 lysozyme powders were denatured using mechanical stress ${ }^{10}$ and $\gamma$-radiation ${ }^{29}$, and the researchers 261 attributed these changes to a decrease in the $\alpha$-helix with an increase in the $\beta$-sheet and disordered 262 structures. However, in the case of the thermally denatured powders, a new Raman band at 1787 $263 \mathrm{~cm}^{-1}$ appeared, and its intensity decreased by raising the water content (Figure 4, spectra dC, dD 264 and $\mathrm{dE}$ ), and the peak disappeared at water content $\geq 12 \% \mathrm{w} / \mathrm{w}$ (Figure 4, spectra $\mathrm{dA}$ and $\mathrm{dB}$ ). 
265 Therefore, the appearance of this peak at $1787 \mathrm{~cm}^{-1}$ relates to the nature of lysozyme molecules' 266 movement during the thermal unfolding transition and this movement depends heavily on the 267 amount of hydration degree of the powders. The new band at $1787 \mathrm{~cm}^{-1}$ could be assigned to the 268 stretching of free hydrogen bonding carbonyl group. As the line of amide I at wavenumbers $\geq 1685$ $269 \mathrm{~cm}^{-1}$ is expected to be assigned to the disordered secondary structure without hydrogen bonding. ${ }^{27}$

270 It can be concluded that the higher the water content in lysozyme powders during unfolding 271 transition, the higher the freedom for lysozyme molecules to move and saturate the free carbonyl 272 groups of the peptide bonds resulted from the unfolding transition. At water content $\geq 12 \% \mathrm{w} / \mathrm{w}$, 273 the lysozyme molecules have enough freedom to move and saturated all the free carbonyl groups, 274 and this explains the disappearance of the Raman band at $1787 \mathrm{~cm}^{-1}$. This finding agrees with 275 previous results stating that at water content between 11 and $21 \% \mathrm{w} / \mathrm{w}$, lysozyme possess a 276 glasslike dynamic transition from rigid to flexible state due to the mobility increase of the structural 277 units. $^{30}$

278 Therefore, the shape of amide I Raman band, the position and intensity of amide III Raman 279 band, and the intensity of the Raman band at $\sim 1787 \mathrm{~cm}^{-1}$ of the thermally denatured powders 280 depend on the water content of their original native powders. This indicates that the molecular 281 structure of the thermally denature lysozyme depends on its hydration degree.

282 This folding-unfolding transition diagram of lysozyme was obtained using sealed pans in 283 order to ensure mass conservation of the water-lysozyme system. Therefore, it was determined at 284 constant volume but variable pressure. Fortunately, in our case, we capsulated the sealed pans at 285 atmospheric pressure ( 0.1MPa), and according to the general gas law, the increase in the pressure 286 due to the temperature increase from 298 to $473 \mathrm{~K}$ inside the hermetically sealed pans would reach 
$287 \sim 0.2 \mathrm{MPa}$. This pressure values have a negligible role in the lysozyme denaturation. It was 288 confirmed that lysozyme is denatured when pressure is at much higher values $>500 \mathrm{MPa}^{31}$

\section{CONCLUSIONS}

These results allowed us to derive an equation correlating $T_{m}$ of lysozyme with its 293 hydration degree. $T_{m}$ exponentially decreased when the hydration degree increased, and it did not

294 significantly decrease further after hydration degree reaching a value similar to hydration shell of 295 the lysozyme. We used the derived equation to draw the folding-unfolding transition diagram of 296 lysozyme. This diagram will be useful to process lysozyme at predetermined conditions of 297 temperature and water content without denaturation. The molecular conformation of the thermally 298 denatured lysozyme was found to be dependent on the hydration degree of the original native 299 powders. A new Raman band at $1787 \mathrm{~cm}^{-1}$ appeared in the spectra of the thermally denatured 300 lysozyme, and its intensity reversibly correlated with the water content and it disappeared at the 301 water content $\geq 12 \% \mathrm{w} / \mathrm{w}$.

302

\section{AUTHOR INFORMATION}

304 Corresponding Author

305 *E-mail: mam2014uk@gmail.com

$306 \quad$ Notes

307 The authors declare no competing financial interest. 

gratefully acknowledges CARA (Stephen Wordsworth and Ryan Mundy) and University of Bradford for providing an academic fellowship.

\section{REFERENCES}

(1) Ji, C.; Mei, Y. Some practical approaches to treating electrostatic polarization of proteins. Acc. Chem. Res. 2014, 47, 2795-2803.

(2) Dhindsa, G. K.; Tyagi, M.; Chu, X. Q. Temperature-dependent dynamics of dry and hydrated beta-casein studied by quasielastic neutron scattering. J. Phys. Chem. B 2014, 118, 10821-10829. (3) Frauenfelder, H.; Chen, G.; Berendzen, J.; Fenimore, P. W.; Jansson, H.; McMahon, B. H.; Stroe, I. R.; Swenson, J.; Young, R. D. A unified model of protein dynamics. Proc. Natl. Acad. Sci. U.S.A. 2009, 106, 5129-5134.

(4) Broadhead, J.; Rouan, S. K.; Hau, I.; Rhodes, C. T. The effect of process and formulation variables on the properties of spray-dried beta-galactosidase. J. Pharmacol. Pharmacother. 1994, 46, 458-467.

(5) Wang, W. Lyophilization and development of solid protein pharmaceuticals. Int. J. Pharm. 2000, 203, 1-60.

(6) Miyazaki, Y.; Matsuo, T.; Suga, H. Low-temperature heat capacity and glassy behavior of lysozyme crystal. J. Phys. Chem. B 2000, 104, 8044-8052.

(7) Panagopoulou, A.; Kyritsis, A.; Aravantinou, A. M.; Nanopoulos, D.; Serra, R. S. I.; Ribelles, J. L. G.; Shinyashiki, N.; Pissis, P. Glass transition and dynamics in lysozyme-water mixtures over wide ranges of composition. Food Biophys. 2011, 6, 199-209.

(8) Bell, L. N.; Hageman, M. J.; Muraoka, L. M. Thermally induced denaturation of lyophilized bovine somatotropin and lysozyme as impacted by moisture and excipients. J. Pharm. Sci. 1995, 84, 707-712.

(9) Kocherbitov, V.; Latynis, J.; Misiunas, A.; Barauskas, J.; Niaura, G. Hydration of lysozyme studied by Raman spectroscopy. J. Phys. Chem. B 2013, 117, 4981-4992.

(10) Mohammad, M. A.; Grimsey, I. M.; Forbes, R. T. Mapping the solid-state properties of crystalline lysozyme during pharmaceutical unit-operations. J. Pharm. Biomed. Anal. 2015, 114, 176-183.

(11) Maroufi, B.; Ranjbar, B.; Khajeh, K.; Naderi-Manesh, H.; Yaghoubi, H. Structural studies of hen egg-white lysozyme dimer: comparison with monomer. Biochim. Biophys. Acta 2008, 1784, 1043-1049.

(12) Yano, Y. F. Kinetics of protein unfolding at interfaces. J. Phys. Condens. Matter 2012, 24, 503101-503117.

(13) Wu, Z.; Cui, Q.; Yethiraj, A. Driving force for the association of hydrophobic peptides: the importance of electrostatic interactions in coarse-grained water models. J. Phys. Chem. Lett. 2011, $349 \quad 2,1794-1798$. 
(14) Tilton, R. F. Jr.; Kuntz, I. D. Jr.; Petsko, G. A. Cavities in proteins: Structure of a metmyoglobin xenon complex solved to 1.9 .ANG. Biochemistry 1984, 23, 2849-2857. (15) Dwyer, J. J.; Gittis, A. G.; Karp, D. A.; Lattman, E. E.; Spencer, D. S.; Stites, W. E.; Garcíapenetration. Biophys. J. 2000, 79, 1610-1620.

(16) Pitera, J. W.; Falta, M.; Van Gunsteren, W. F. Dielectric properties of proteins from simulation: the effects of solvent, ligands, pH, and temperature. Biophys. J. 2001, 80, 2546-2555. (17) Kocherbitov, V.; Arnebrant, T. Hydration of thermally denatured lysozyme studied by sorption calorimetry and differential scanning calorimetry. J. Phys. Chem. B 2006, 110, 1014410150.

(18) Vinh, N. Q.; Allen, S. J.; Plaxco, K. W. Dielectric spectroscopy of proteins as a quantitative experimental test of computational models of their low-frequency harmonic motions. J. Am. Chem. Soc. 2011, 133, 8942-8947.

(19) Knab, J.; Chen, J. Y.; Markelz, A. Hydration dependence of conformational dielectric relaxation of lysozyme. Biophys. J. 2006, 90, 2576-2581.

(20) Bellavia, G.; Paccou, L.; Achir, S.; Guinet, Y.; Siepmann, J.; Hédoux, A. Analysis of Bulk and Hydration Water During Thermal Lysozyme Denaturation Using Raman Scattering. Food Biophys. 2013, 8, 170-176.

(21) Svergun, D. I.; Richard, S.; Koch, M. H. J.; Sayers, Z.; Kuprin, S.; Zaccai, G. Protein hydration in solution: experimental observation by x-ray and neutron scattering. Proc. Natl. Acad. Sci. U.S.A. 1998, 95, 2267-2272.

(22) Kuntz, I. D. Hydration of macromolecules. III. Hydration of polypeptides. J. Am. Chem. Soc. 1971, 93, 514-516.

(23) Choudhuri, J. R.; Chandra, A. An ab initio molecular dynamics study of the hydrogen bonded structure, dynamics and vibrational spectral diffusion of water in the ion hydration shell of a superoxide ion. Chem. Phys. 2014, 445, 105-112.

(24) Blumlein, A.; McManus, J. J. Reversible and non-reversible thermal denaturation of lysozyme with varying $\mathrm{pH}$ at low ionic strength. Biochim. Biophys. Acta. Proteins and Proteomics 2013, 1834, 2064-2070.

(25) Chen, P.; Seabrook, S. A.; Epa, V. C.; Kurabayashi, K.; Barnard, A.S.; Winkler, D. A.; Kirby, J. K.; Ke, P. C. Contrasting effects of nanoparticle binding on protein denaturation. J. Phys. Chem. C 2014, 118, 22069-22078.

(26) Yu, T. J.; Lippert, J. L.; Peticolas, W. L. Laser Raman studies of conformational variations of poly-L-lysine. Biopolymers 1973, 12, 2161-2176.

(27) Li-Chan, E. C. Y. The applications of Raman spectroscopy in food science. Trends. Food. Sci. Tech. 1996, 7, 361-370.

(28) Yu, N. T.; Liu, C. S. Laser Raman Spectra of Crystalline and Aqueous Glucagon. J. AM. Chem. Soc. 1972, 94, 5127-5135.

(29) Torreggiani, A.; Tamba, M.; Manco, I.; Faraone-Mennella, M. R.; Ferreri, C.; Chatgilialoglu, C. Radiation damage of lysozyme in a biomimetic model: some insights by Raman spectroscopy. J. Mol. Struct. 2005, 744, 767-773.

(30) Kocherbitov, V.; Arnebrant, T.; Söderman, O. Lysozyme-water interactions studied by sorption calorimetry. J. Phys. Chem. B 2004, 108, 19036-19042.

(31) Hédoux, A.; Guinet, Y.; Paccou, L. Analysis of the mechanism of lysozyme pressure denaturation from Raman spectroscopy investigations, and comparison with thermal denaturation. J. Phys. Chem. B 2011, 115, 6740-6748. 
$T_{m}=-36.4 \ln N+545.7$ when $N \leq N^{S}$

$$
T_{m} \cong T_{m}^{S} \text { when } N \geq N^{S}
$$

$T_{m}$ : Apparent denaturation temperature of lysozyme.

$\mathrm{N}$ : Ratio of water molecules to lysozyme molecules.

\section{S: Superscript refers to saturated} water-lysozyme state. 